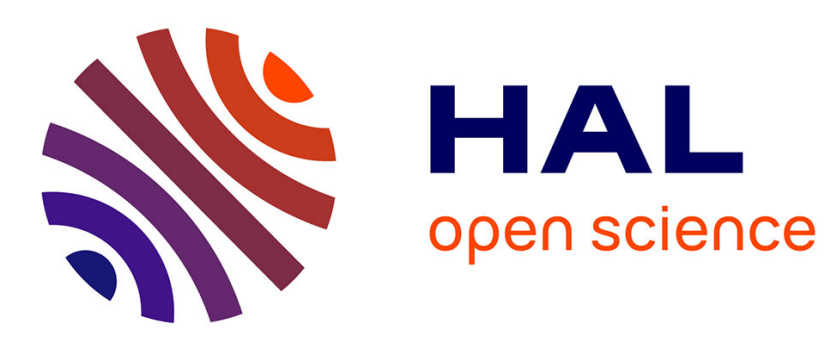

\title{
Koizumi Jun'ichirô superstar de la vie politique japonaise
}

Eric Seizelet

\section{To cite this version:}

Eric Seizelet. Koizumi Jun'ichirô superstar de la vie politique japonaise. Le Temps des médias. Revue d'histoire, 2008, 10, pp.115-128. halshs-00293204

\section{HAL Id: halshs-00293204 https://shs.hal.science/halshs-00293204}

Submitted on 3 Jul 2008

HAL is a multi-disciplinary open access archive for the deposit and dissemination of scientific research documents, whether they are published or not. The documents may come from teaching and research institutions in France or abroad, or from public or private research centers.
L'archive ouverte pluridisciplinaire HAL, est destinée au dépôt et à la diffusion de documents scientifiques de niveau recherche, publiés ou non, émanant des établissements d'enseignement et de recherche français ou étrangers, des laboratoires publics ou privés. 


\section{Koizumi Jun'ichirô superstar de la vie politique japonaise. Par \\ Eric Seizelet ${ }^{*}$}

\section{Introduction}

En avril 2001, Koizumi Jun'ichirô accède à la tête du gouvernement japonais avec $87,1 \%$ d'opinions favorables, soit l'un des taux de popularité les plus élevés de l'après-guerre. Il inaugure ainsi un long gouvernement de cinq ans, voué à la mise en place de réformes structurelles bousculant les lobbies et les pratiques politiques fondées sur les équilibres factionnels à l'intérieur du parti dominant, le PLD. Lorsqu'il quitte son mandat en septembre 2006, c'est encore avec un taux de popularité supérieur à $40 \%$. Koizumi Jun'ichirô se distingue ainsi de ces prédécesseurs au moins par trois caractéristiques : 1) il commence son double mandat de président du parti majoritaire et de Premier ministre avec l'un des taux de soutien les plus élevés de l'après-guerre, preuve s'il en était que les attentes de la population en faveur du changement étaient grandes après les maladresses de son prédécesseur Mori Yoshirô ; 2) il termine son mandat avec un taux de soutien relativement confortable pour un chef de gouvernement japonais, échappant à la malédiction qui veut que le Premier ministre connaisse un déclin constant de popularité. Certes Koizumi Jun'ichirô n'a pas totalement échappé à ce phénomène d'érosion du pouvoir, puisque durant ce « quinquennat », le taux de soutien a chuté de plus de moitié. Mais après cinq années de responsabilité - une durée exceptionnelle pour un chef de gouvernement nippon - son score, à l'aulne de ses prédécesseurs, demeure plus que confortable. Et lorsqu'il quitte ses fonctions en septembre 2006, c'est auréolé du triomphe remporté de haute lutte aux élections générales de septembre 2005 au cours desquelles la majorité gouvernementale PLD-Kômeitô avait raflé plus des deux-tiers des sièges à la chambre des Représentants, jouissant d'un taux de popularité moyen de $56 \%$ sur toute la durée de son administration, parmi les plus élevés de l'après-guerre ; 3) il inaugure enfin un style politique nouveau où il capitalise sur sa personne les effets de la réforme politique et administrative que l'un de ses prédécesseurs et rivaux, M. Hashimoto Ryûtarô, avait instituée en 1999 afin de renforcer les capacités de planification du Cabinet, avec une présidentialisation de la fonction de Premier ministre accélérant le phénomène de personnalisation du pouvoir. Mais le « succès » de Koizumi Jun'ichirô n' aurait été rien sans la rencontre d'un homme et d'une conjoncture. Il fait souffler un vent nouveau dans un Japon en proie à des doutes existentiels, déstabilisé par un système politique à bout de souffle, l'obsolescence d'un modèle socio-économique mis à mal par une décennie de crises et une image internationale encore floue. Par ailleurs, l'ascension de Koizumi Jun'ichirô ne fait que confirmer l'exposition grandissante des hommes politiques aux médias, principalement audiovisuels, depuis le milieu des années 80 , la montée en puissance de l'infotainement, c'està-dire l'investissement des émissions de variétés par la politique, à travers les talk shows et les wide shows les plus courus tels que Sunday Project de la chaîne Asahi, le rôle de ces nouveaux espaces de communication dans la construction de la notoriété des politiciens, et le poids de l'image dans les stratégies électorales des élus, surtout depuis que la réforme de 1994 en introduisant un scrutin uninominal à un tour dans des circonscriptions à siège unique, a accéléré la chasse par les partis aux candidats célèbres, les talento, issus des milieux du spectacle ou du sport ${ }^{1}$.

\footnotetext{
* Directeur de l'Institut d'Asie Orientale, Université de Lyon, Lyon, F-69003, France.

${ }^{1}$ Masaki Taniguchi, "Changing Media, Changing Politics in Japan », Japanese Journal of Political Science, mars 2007, vol. $8, n^{\circ} 1$, pp. 147-166.
} 
Qualifié souvent de «populiste », l'ancien chef du gouvernement l'est assurément par certains côtés : tout au long de son mandat, il a stigmatisé les "forces de la réaction » qui s'opposaient, à l'intérieur de la bureaucratie et de son propre parti aux réformes structurelles. Il rompait ainsi délibérément non seulement avec l'hypocrisie d'une gestion " consensuelle » et « harmonieuse » des rapports de force à l'intérieur du parti dominant ${ }^{2}$. Populiste aussi le souhait de s'entourer de personnalités au profil atypique et médiatique. Son « compagnonnage politique » avec Mme Tanaka Makiko, en début de mandat, fut d'abord un coup médiatique : il signalait le ralliement de la fille d'un ancien chef du gouvernement, Tanaka Kakuei, qui fut longtemps « faiseur de rois » au Japon et ennemi juré de Fukuda Takeo, mentor politique de Koizumi. Promue ministre des Affaires Etrangères, elle y déploya un zèle peu commun à s'aliéner sa propre administration en multipliant dans les médias les déclarations intempestives et les ingérences dans les nominations au sein du ministère. Lorsque le Premier ministre, lassé de ses frasques, décida de s'en séparer au début 2002, sa popularité chuta aussitôt dans les sondages, principalement auprès de l'électorat féminin : pour une partie de l'opinion, Mme Tanaka n'était pas victime de son incompétence mais de l'arrogance de la bureaucratie. Pour se tirer de ce trou d'air, Koizumi multiplia les apparitions à la télévision: donnant le coup d'envoi du match amical entre le Japon et le Costa-rica, figurant en gastronome éclairé dans un programme de cuisine japonaise, participant à des campagne de sensibilisation des écoliers à la sécurité routière... Même déconvenue avec le flamboyant Horie Takafumi, le charismatique patron du portail internet Livedoor : prototype de ces jeunes entrepreneurs ambitieux issus de la «nouvelle économie », favori des plateaux de télévision pour ses apparitions provocantes et son mode de vie extravagant, cultivant un mépris souverain pour l'establishment des milieux d'affaires qui le lui rend bien, Koizumi n'hésite pas à s'afficher à ses côtés, parce qu'il est le symbole de la réussite d'un nouveau Japon sortant de l'ornière de la crise, au point de lui demander de se présenter à Hiroshima en septembre 2005 lors des élections générales. Rattrapé par les « affaires », Horie sera arrêté en janvier 2006 et condamné en mars 2007 à deux ans et demi de prison par la justice pour manipulation de cours de bourse. Populiste aussi, la propension du chef du chef du gouvernement à la dramatisation et à la polarisation des enjeux au prix d'une simplification et d'une radicalisation du discours politique. Il concourt ainsi à la stigmatisation de l'adversaire, à la publicisation et à l'élévation de la conflictualité politique, transformant les consultations électorales en plébiscite. Populiste enfin la propension à investir le politique d'une dimension intuitive et émotionnelle très forte, dans laquelle le contenu même du message s'efface devant le contenant, l'action devant la communication, le propos devant la cible ${ }^{3}$.

\footnotetext{
${ }^{2}$ C'était vrai non seulement pour la sélection de son premier Cabinet qui ne tenait pas compte des dosages factionnels au sein du PLD mais aussi en 2005, lors de la dissolution consécutive au rejet par la chambre des Conseillers du projet de loi de privatisation de la Poste. Le Premier ministre mit au point la stratégie de punition des parlementaires PLD qui avaient voté contre la réforme, en refusant de leur accorder l'investiture et en leur envoyant des « tueurs », sekkaku, dans leur propre circonscription, c'est-à-dire en y parachutant des candidatures rivales pour les faire échouer, ce qui sera le cas dans 14 circonscriptions sur 37. Le fait que certaines de ces « tueurs » aient été en réalité des « tueuses » ajouta encore au caractère provocateur de cette initiative : Naofumi Fujimura, « The Power Relationship between the prime Minister avd Ruling Party Legislators : The Postal Service Privatization Act of 2005 in Japan », Japanese Journal of Political Science, août 2007, vol. 8, n², pp. 233-261. Egalement, Ikuo Kabashima et Gill Steel, «The Koizumi Revolution», PS : Political Science \& Politics, 2007, n 40 , pp. 79-84.

${ }^{3}$ Sur ces différents points: Ôtake Hideo, Koizumi Jun'ichirô popyurizumu no kenkŷ, recherches sur le populisme de Koizumi Jun'ichirô, Tôkyô, 2006, Tôyô keizai shimpôsha, p. 251 et s. ; Machida Tetsu, Nihon no yûsei, tokihanatareta kyojin, La politique des postes et télécommunications, le géant « libéré », Tôkyô, 2005 , Nihon keizai shimpôsha, p. 200 et s.
} 
Koizumi Jun'ichirô est apparu sous les feux de la rampe politiques précédé de la réputation d'un « homme bizarre », de « loup solitaire », voire d'un « excentrique ». D'abord par son apparence physique: adepte des costumes clairs et des cravates de couleur, voire flashy, il détonne dans une classe politique dont les costumes et accessoires sombres sont un signe de respectabilité. Son allure jeune - jeuniste pour ses détracteurs - et décontractée, son sens de la répartie et ses propos à l'emporte-pièce tranchent sur le maintien compassé et affecté de ses collègues de la Diète ${ }^{4}$. Sa « crinière » abondante et soigneusement permanentée, popularise sur tous les écrans son visage de «Lion ». Son goût affiché pour le hard rock et la musique techno est peu commun chez un homme proche de la soixantaine. En décembre 2005 , on le voit se rendre à ses bureaux en Segway, véhicule électrique improbable à deux roues offert par le président américain, et interdit de circulation à Tôkyô pour cause de... dangerosité. Politiquement, il passe pour un marginal : il ne figurait parmi les caciques du PLD; sa base à l'intérieur même du PLD, où il n'a jamais fondé de véritable clientèle politique restait faible. Pourtant, à la différence des populistes qui, parce qu'ils sont à l'extérieur du système, s'emploient à le torpiller auprès de l'opinion en jouant les hommes nouveaux non compromis par les prébendes du pouvoir, le Premier ministre était en fait un pur produit de la nomenclatura nippone : c'était tout d'abord un héritier, car son père et son grand-père avaient été respectivement directeur de l'agence de Défense et vice-président de la chambre des Représentants, comme beaucoup de parlementaires PLD. Elu à dix reprises à la chambre des Représentants en 2001, il avait été successivement vice-ministre gouvernemental, seimujikan, des Finances, puis trois fois ministre, de la Santé dans les gouvernements Takeshita et Hashimoto, des Postes dans le Cabinet Miyazawa, et secrétaire général de la faction Mori. A la Santé, il se distingue cependant en faisant de la lutte contre le tabagisme un enjeu de santé publique au grand dam du PLD et de l'industrie du tabac, tout en plaidant, « à titre personnel » pour l'autorisation du viagra, jugée plus importante que la lutte contre la dioxine... Une carrière honorable, sans plus, mais suffisante pour en faire un bon connaisseur des rouages du PLD et de ses clans, mais qui ne pouvait en aucun cas le faire passer pour un " homme nouveau », même si tous les observateurs reconnaissent qu'il a toujours pris soin d'éviter d'être happé par le jeu des factions et qu'il s'est tenu à l'écart des scandales qui ont miné ses prédécesseurs. Son ascension même n'a pas été fulgurante puisqu'il lui a fallu s'y reprendre à trois reprises pour accéder au poste de président du PLD qui lui ouvrait automatiquement l'accès aux fonctions de chef du gouvernement.

\section{Koizumi Jun'ichirô et la mise en scène du politique}

Koizumi Jun'ichirô n'a pas été une création médiatique, mais il est certain que son accession à la tête du PLD, puis au poste de Premier ministre, a été facilitée par une couverture médiatique plus importante, un talent honnête d'orateur et une maîtrise plus grande de l'outil télévisuel qui joua un rôle déterminant dans une compétition pour le pouvoir beaucoup plus ouverte, et dans laquelle Koizumi apparaissait de prime abord comme outsider ${ }^{5}$.

Car Koizumi Jun'ichirô est également un communicateur : ses déplacements sont habilement mis en scène, en particulier par son assistant parlementaire de toujours, M. Iijima

\footnotetext{
${ }^{4}$ Du coup, au début de l'administration Koizumi, les retransmissions télévisées des débats à la Diète par la NHK connaissent un taux d'audience supérieur de plus de deux points qu'à l'ordinaire, soit une moyenne se situant entre $4,5 \%$ et $6,5 \%$, avec des pics à $13,1 \%$ : des résultats à l'époque supérieurs à ceux des talk ou wide shows les plus populaires. Il en est de même de la progression des souscriptions à la chaine parlementaire sur le câble.

${ }_{5}^{5}$ Ikuo Kabashima, Gille Steel, «How Junichiro Koizumi seized the leadership of Japan's Liberal Democratic Party », Japanese Journal for Political Science, 2007, vol. 8, ${ }^{\circ} 1$, pp. 95-114.
} 
Isao, surnommé par les médias le « Raspoutine de la Résidence du Premier ministre », et que les médias américains comparent à Carl $\mathrm{C}$. Rove qui fut longtemps considéré comme le double du président Bush. Cet ordonnateur de la stratégie médiatique du Premier ministre entretient cependant avec la presse une attitude ambiguë : il n'hésite pas à saisir la justice pour diffamation lorsque des hebdomadaires populaires tels que Shukan gendai, Shukan bunshu, ou la chaine de télévision Fuji telebi, font état de son rôle occulte dans la politique du gouvernement à l'égard de la Corée du Nord, dans la sélection des membres du Cabinet ou le qualifient «d'ombre du Premier ministre », Sôri no kage. Mais en même temps, c'est lui qui révolutionne la communication de Koizumi : privilégier le visuel sur l'écrit, et notamment les wide shows, et dans la presse écrite, les tabloïds plutôt que les journaux traditionnels, le sens de la formule plutôt que les longs discours, occuper le terrain des médias par deux points de presse quotidiens, dont l'un en présence des caméras. C'est d'ailleurs à cette fin qu'il orchestre, pour le compte du chef du gouvernement la "mise en scène » de l'entrée du Premier ministre au Kokugikan de Tôkyô, pour la remise du prix du Premier ministre lors du tournoi de printemps de mai 2001. Evènement triplement médiatique : d'abord il est rare que le chef du gouvernement se déplace en personne pour la remise de ce trophée; en second lieu, au lieu de la lecture convenue du diplôme qui accompagne la remise du prix, le Premier ministre félicita chaleureusement le vainqueur du tournoi donnant à cette cérémonie une spontanéité et une chaleur qu'elle n'avait pas; ensuite en associant à cette mise en scène la presse sportive qui n'avait pas normalement d'accès direct au chef du gouvernement : du coup les grands quotidiens sportifs tels que Gekkan spôtsu, Spôtsu nippon et Sankei spôtsu furent conviés à s'accréditer auprès de la presse en charge de la résidence officielle du Premier ministre. Il sait user de la télévision et des médias pour marteler à l'envi son programme réformiste et jouer ainsi l'opinion contre les féodalités bureaucratiques et partisanes. Il tient un discours politique simple - simpliste disent ses adversaires - mais sait, quand arrivent les échéances électorales, dramatiser les enjeux et payer de sa personne en parcourant les estrades électorales. En bref, il transforme la vie politique en «théâtre» dont il est le principal producteur et metteur en scène ${ }^{6}$.

Koizumi Jun'ichirô n'est certes pas le premier homme politique japonais à avoir compris le rôle de l'image en politique : l'un de ses prédécesseurs, Tanaka Kakuei, avait été dans la première moitié des années 1970 le favori des médias, avant de sombrer dans le scandale de l'affaire Lockheed. Il avait été très populaire auprès de l'opinion pour son style et sa faconde populaire. Self made man, Tanaka, surnommé le «bulldozer », qui n'avait pas fréquenté l'Université et n'appartenait pas de ce fait à l'élite bureaucratique du pays, avait donné un temps l'illusion que l'homme de la rue pouvait accéder aux plus hautes fonctions. Nakasone Yasuhiro, dans les années 1980, avait contribué à présidentialiser à l'international l'image du Premier ministre en entretenant une relation privilégiée avec le président américain Ronald Reagan. Hosokawa Morihiro en 1993-1994, avait été l'un des plus jeunes chefs de gouvernement de l'après-guerre. D'un physique avenant, comparé souvent à John F. Kennedy pour son sourire éclatant, cet aristocrate, familièrement dénommé tono sama, Votre Seigneurie, avait joui d'un taux de popularité élevé avant d'être rattrapé par les scandales politico-financiers qui avaient terni son image de «Monsieur Propre ». Déjà à cette époque se mettaient en place les ingrédients nipponisés de la "politique spectacle » : l'omniprésence de la télévision et en particulier des talk shows et autres wide shows qui mêlent actualités, faits divers, potins du gotha, principalement à destination des femmes au foyer, mais où se conjuguent les intérêts bien sentis des hommes politiques, soucieux d'entretenir leur image

\footnotetext{
${ }^{6}$ Philippe Pons, « Le théâtre Koizumi », Le Débat, mars-avril 2006, pp. 39-48. Cette expression a été utilisée par les observateurs japonais eux-mêmes, Koizumi gekijô, d'abord de façon péjorative, puis reprise par les partisans du Premier ministre?
} 
auprès de leurs électeurs, et ceux des médias rivés aux taux d'audience et aux parts de marché pour attirer et conserver la faveur des annonceurs. Les effets de cette banalisation du politique sont difficiles à cerner, mais, si l'on en croit les sondages, la télévision, plus que la presse écrite est devenue la principale source d'information pour $82,4 \%$ des Japonais (2003); elle concourt à propager une image différente des hommes politiques en stimulant les réflexes d'empathie. Les animateurs de ces émissions leur attribuent même des vertus pédagogiques : elles intéresseraient à la politique des électeurs qui auraient tendance à la fuir parce qu'elles la rendraient plus accessible et plus compréhensible aux yeux du public. De fait, la popularité de Koizumi Jun'ichirô doit beaucoup au marais des électeurs sans identification partisane, les mutô-ha qui représentaient plus de $50 \%$ de l'électorat, nombreux dans les grandes agglomérations

\section{L'obsession ambiguë de la proximité}

La popularité du Premier ministre Koizumi tient non seulement à un style politique décalé par rapport à ses prédécesseurs, mais aussi à une personnalité « proche » à laquelle les Japonais pouvaient plus facilement s'identifier. Toutes les enquêtes d'opinion insistent sur cette image de proximité : il est «épanoui », « sympathique », "doté du sens de l'humour », " chaleureux », " simple ». En d'autres termes, il est l'image inversée du politicien japonais traditionnel. Cette proximité va d'ailleurs de paire avec une autre qualité reconnue au Premier ministre : son dynamisme (il est « habile», « entreprenant» et « réformiste » ${ }^{7}$. Elle s'accompagne d'ailleurs d'une gestuelle et d'une mobilité du visage plus riche que celle de ses collègues qui contribuent à rendre le Premier ministre moins guindé, plus "cool» et spontané. Il pratique également le bain de foule, peu courant chez les hommes politiques japonais au moins pour trois raisons : policières par souci de sécurité, politiques du fait du discrédit général qui entoure la classe politique peu propice à ce genre de manifestations et culturelles en raison des usages sociaux rétifs à l'effusion publique de contacts physiques réservés normalement à la sphère privée.

L'usage des nouvelles technologies de la communication et de l'information joua également un rôle important dans la construction de l'image du Premier ministre comme star de la politique. En mai 2001, Koizumi Jun'ichirô lança son propre fan site, bientôt connecté à celui de son fils Kôtarô lorsque ce dernier fera ses débuts dans le show-business, et surtout un email-magazine permettant de recevoir gratuitement, chaque semaine, sur PC, PDA et téléphone mobile, une lettre interactive d'information, habilement présentée sous la dénomination "Cœur de Lion : un message du Cabinet du Premier ministre », allusion à la coiffure «léonine » de Koizumi et un clin d'œil à l'une des chansons du groupe SMAP, un boys band particulièrement populaire du moment. En mars 2004, 1,7 millions d'internautes y étaient abonnés. Pour la première fois, le Japon faisait l'expérience à l'échelon national, de la cyberdémocratie : Koizumi Jun'ichirô y expose ses goûts personnels, en matière de musique, de lecture et de sports en fonction de l'actualité du moment, ses doutes, les contraintes de la fonction de chef de gouvernement, sa volonté d'y faire face et d'expliquer sa politique à la population. Les abonnés de leur côté sont invités à écrire au Premier ministre pour lui faire part de leurs suggestions et critiques afin d'améliorer le programme du gouvernement. On y trouve aussi naturellement des sondages d'opinion, et des enquêtes sur les produits dérivés, les Koizumi goods. L'usage combiné d'internet et de la télévision permettait ainsi une

\footnotetext{
${ }^{7}$ Voit par exemple, Kobayashi Yoshiaki, « Sôzô ni ataranu hakai. Sôkatsu koizumi seiken no yonenhan », La destruction non constructive : regards sur les quatre ans et demie de gouvernement Koizumi, Sekai, $\mathrm{n}^{\circ} 743$, septembre 2005, pp. 151-152.
} 
communication à double détente : de masse, unilatérale et brève à la télévision, plus longue, personnalisée et interactive sur le web.

Cette expérience de " démocratie en ligne » ou cathodique, sous les dehors attrayants des nouvelles technologies participe elle aussi de l'impact du populisme sur le système politique nippon. Rien ne permet en effet d'affirmer que les décisions du gouvernement aient été réellement affectées par les clics de souris des citoyens. Mais il en ressort deux principaux aspects : 1) le contournement des modes, structures de communication entre les élites et le peuple par l'instauration d'un dialogue direct et « intime » entre le gouvernement et la population court-circuitant les instances traditionnelles de médiation et d'expression du pouvoir - partis ou médias - ou les instrumentalisant au service de buts politiques ; 2) la densification de l'image dans laquelle le message spécifiquement politique est dilué par une communication ciblée sur la valorisation de la personnalité de l'homme politique. Mais cette stratégie alimente également un double soupçon : d'une part la mise à contribution des fonds publics dans la propagande et la construction de l'image du chef du gouvernement ${ }^{8}$; d'autre part, le contournement par le biais de l'internet et des émissions « grand public » des règles très strictes qui encadrent au Japon les campagnes électorales. Elle a également des effets pervers en ce qu'elle génère de nouveaux conformismes : il devient impossible à l'homme politique de modifier l'image sur laquelle sa popularité s'est fondée et elle amplifie les réactions de rejet à l'encontre de la frange de l'opinion publique qui n'adhère pas au « culte » Koizumi. Les discours et positions alternatives critiques deviennent inaudibles à la télévision ou provoquent des réactions violentes de « fans » qui ne comprennent pas que l'on puisse s'en prendre, publiquement, à leur « idole $»^{9}$.

\section{Les produits dérivés ou la marchandisation du politique}

Si l'apparition des hommes politiques dans des émissions de variétés n'était pas chose nouvelle au Japon, principalement depuis 1993, la mise sur orbite de l' « homme bizarre » se solde par l'arrivée sur le marché de toute une série de « produits » qui concourent à propulser la Koizumimania à des niveaux jusque-là inégalés: en juin 2001, le PLD lance un concours de mascotte. C'est le projet d'une jeune femme de 24 ans originaire du département d'Aichi qui sera retenu par le Premier ministre parmi 226 propositions. La mascotte, Shishirô, est un lion en peluche, à l'effigie du Premier ministre, avec un cœur rouge sur la poitrine. Le lion renvoie à la «crinière » du Premier ministre mais aussi à une discrète allusion familiale : le père de Koizumi, Matajirô, avait été ministre des Transports et des Communications sous le Cabinet dirigé par Hamaguchi Osachi surnommé «Le Lion ». Le terme même de Shishi signifie également patriote, et qualifie généralement quelqu'un de dévoué à une cause, ce qui correspond bien à l'image de détermination du Premier ministre. Enfin, les posters, affiches, badges, à l'effigie du chef du gouvernement, s'arrachent au siège du parti libéral-démocrate qui n'avait jamais enregistré pareille affluence. Collégiennes et lycéennes ne sont pas les dernières à succomber à l'attraction de ces produits mode qui font tendance : en cinq jours, au début du mois de mai 2001, 235000 posters sont ainsi vendus, à perte d'ailleurs, au QG du PLD, pour atteindre 1 million d'exemplaires un an plus tard. On peut également y trouver des serviettes, des chopes, des breloques pour téléphone portable, des chemises, des tee-shirts, des assiettes assorties d'une calligraphie du Premier ministre, des porte-clefs, des cartes

\footnotetext{
${ }^{8}$ Rappelons par exemple que la construction et l'entretien du site web du Premier ministre a coûté en moyenne 198 millions de yens par an au contribuable, l'email-magazine 156 millions et que les dépenses de communication du siège officiel du Premier ministre ont atteint sur cinq ans 2,4 milliards de yen.

${ }^{9}$ Voir par exemple, Uesugi Takashi, Koizumi no shôri, media no haiboku, la victoire de Koizumi et la défaite des médias, Tôkyô, 2006, Sôshisa.
} 
téléphoniques, des calendriers, le tout compacté dans des "dream packs » pour toutes les bourses, allant de 200 à 4200 yen. Les boutiques de souvenirs à la Diète connaissent un regain d'activité : on y vend, pour 350 yens, des tasses à thé à l'effigie des Premiers ministres japonais qui se sont succédé depuis 1885. Celle de Koizumi Jun'ichirô devient rapidement un « must ». Dans les lieux publics fréquentés de Tôkyô et en particulier dans les galeries et échoppes attenantes au sanctuaire de Yasukuni, haut lieu controversé du shintô d'Etat et de visites du Premier ministre qui ne l'étaient pas moins, on pouvait trouver des pâtisseries japonaises avec la silhouette du Premier ministre. A Yokosuka, le fief de Koizumi dans la banlieue de Tôkyô, nombre de commerçants arboraient un portrait dessiné ou photographié du chef du gouvernement ; les restaurant affichaient, à divers prix, des menus Jun'chan, le plus souvent assortis d'une exhortation familière au courage et à la ténacité du chef du gouvernement, tandis que les boulangers proposaient des pains ou des gâteaux reproduisant les traits de Koizumi. L'estampille «Jun'chan » n'était pas neutre. Il s'agit d'un diminutif du prénom assorti d'un suffixe de familiarité utilisé pour les enfants en bas âge et les grands parents, que les fans du Premier ministre n'hésitent pas à lancer lors de ses apparitions publiques sur la musique du groupe X-Japan, la même que l'on retrouve dans les publicités télévisées du PLD utilisant le portrait du Premier ministre. L'utilisation de ce diminitif crée un lien affectif, certes fictif, mais immédiat, abolissant la distance entre les locuteurs. Cette inversion de la hiérarchie dans une société où les niveaux de langage structurent l'espace des relations sociales est un signe politique : elle intègre le Premier ministre dans un espace fictif de proximité auquel chacun peut s'identifier : celui de la famille. En juillet 2001, après la victoire du PLD aux élections, un grand magasin de la ville de Yokosuka, le Saikaya, obtient l'autorisation des services du Premier ministre, de commercialiser des Koizumi goods. Le phénomène prend bientôt une ampleur nationale, notamment dans les galeries marchandes des lieux de passage les plus fréquentés comme les gares. Au total, on a pu estimer qu'en 2001, la commercialisation de l'image du Premier ministre a rapporté au PLD, selon les rapports sur le financement politique, 282,14 millions de yens, soit $1 \%$ des revenus du parti, qui peine à protéger la « marque de fabrique », shishirô contre les contrefacteurs. Durant la même période, celle de Hatoyama Yukio, le leader du parti démocrate japonais n'aurait rapporté que 7 millions de yens dans les caisses du PDJ. Passés les effets de mode, au bout d'un an, le " marché » Koizumi se rétrécit : il ne rapporte plus que 6 millions de yen en 2003 et 1,4 seulement en 2005. La saturation du marché, un certain effet de lassitude, le zapping du " consommateur politique », mais aussi l'essoufflement de la popularité du Premier ministre expliquent cette retombée. A partir de janvier 2003, le PLD se trouve contraint de brader les Koizumi goods à $60 \%$ ou $70 \%$ de leur prix de revient. Il est vrai que dès le départ on escomptait que la rentabilité politique de ce marché serait bien supérieur à son coût financier. Mais durant les cinq années de pouvoir de Koizumi, les résultats électoraux du PLD suivent une évolution en dents de scie : le comportement de l'électeur-citoyen ne reflète pas, tant s'en faut, les habitudes du consommateur des Koizumi goods. Bien plus, les électeurs sans attaches partisanes qui avaient fait la fortune du PLD en 2001 ont tendance à retourner dans le giron du PDJ à partir de 2002.

\section{La peopolisation indirecte}

Le terme de «peopolisation » peut avoir un double sens. On peut tout d'abord par là une confusion délibérée et mise en scène de la vie publique et de la vie privée des responsables politiques dont ils sont les protagonistes et parfois les victimes plus ou moins consentantes, le Japon ne présente pas encore tout à fait les mêmes dérives que dans d'autres pays occidentaux. Le peopolisation ne se réduit pas aux affaires d'alcôve qui font l'ordinaire des feuilles à scandale et si certains hommes politiques sont tombés à l'instar de l'éphémère 
Premier ministre Uno Seisuke en juin 1989 sur une affaire de mœurs, il s'agissait là d'une " vengeance » d'une maîtresse délaissée. De façon générale, la presse japonaise populaire ne s'était pas vraiment intéressée à la vie privée des hommes politiques, et encore moins de celle $\mathrm{du}$ Premier ministre, parce que ces derniers n'appartenait pas au milieu des people, le geinôkai, où l'exposition de la vie privée est une contrainte plus ou moins intégrée ou acceptée, parce qu'il existait une séparation plus nette, et respectée par les médias, entre les espaces publics et privés, et sans doute aussi parce que la chronique des " affaires » dans lesquelles ils pouvaient être impliqués suffisait amplement à remplir la rubrique des scandales.

Pourtant, des signes avant-coureurs apparaissaient, montrant que ce statu quo était en train de s'effriter : en 2003, la Diète a adopté une loi de protection de la vie privée pour lutter contre l'audace grandissante des tabloïds divulguant des informations sur la vie privée des célébrités et des actions en justice ont été intentées par des hommes politiques. Koizumi Jun'ichirô n'a pas été épargné par des rumeurs touchant sa vie privée ${ }^{10}$. Sa situation familiale et matrimoniale atypique a également contribué à alimenter cette image d'excentricité. Père de trois fils, mais divorcé après quatre ans de mariage en 1982, le Premier ministre n'a jamais rencontré son cadet, Yoshinaga, né trois mois après son divorce, et qui vit écarté des événements familiaux, comme le décès de sa grand-mère. Si les journaux se sont font fait l'écho des frustrations de Yoshinaga, lorsque son père est devenu Premier ministre et de la rancœur de son ex-épouse Mme Miyamoto Kayoko, qui dans la presse internationale, déplorait, elle aussi, d'avoir été séparée de ses deux fils et attribuait à la politique l'échec de son couple, ces doléances n'ont en rien entamé le capital de popularité de ce dernier, dans la mesure où l'attitude de Koizumi était tout à fait conforme aux mœurs en vigueur en cas de divorce. D'autant qu'un contre-feu pouvait être allumé : en obtenant la garde de ses deux autres fils, alors qu'elle est généralement confiée à la mère, il était plus aisé de mettre en valeur la pugnacité d'un père attentif à l'éducation de ses enfants même en cas de séparation. Mais il existait toutefois une lecture différente de l'exposition des déboires familiaux du chef du gouvernement : tester un argumentaire qui aurait pu être utilisé contre lui en cas de d'échec et de chute de popularité. Ayant à plusieurs confirmé publiquement qu'il ne se remarierait jamais, le Premier ministre en tira au moins indirectement un surcroît de popularité : alors que le célibat d'un homme mûr est mal vu au Japon, il est apparu comme un choix de vie et l'indice d'un caractère faisant preuve de la même détermination dans la vie privée que dans l'engagement politique. Naturellement, dans la mesure où le Premier ministre était populaire auprès des femmes, les spéculations allaient bon train. En mai 1992, les tabloïds ont évoqué son nom dans le suicide d'une Geisha à Tôkyô. Plus tard, les assiduités d'une groupie faisant le siège des bureaux officiels du Premier ministre ont brièvement attiré l'attention des médias. Et si on lui prête des aventures, il n'a pas de maîtresse attitrée. En revanche, sa notoriété a eu un impact au moins indirect sur son entourage familial : l'arrivée de Koizumi Jun'ichirô au pouvoir aura eu pour conséquence de stimuler la carrière de son fils aîné Kôtarô : en août 2001 , cet étudiant de 23 ans annonça devant 250 reporters et 26 caméras de télévision son intention de faire carrière dans le spectacle. Depuis, le fils est devenu un top-model pour la marque de vêtement DAKS E1, un habitué des plateaux de télévision et des écrans publicitaires tout en menant une carrière d'acteur. Sans exclure plus tard de faire une carrière politique comme son père...

Le second indice de la peopolisation doit être recherché dans l'adhésion à des codes et à des modes relationnels qui constituent autant d'indices d'appartenance à la communauté de la Jetset et des beautiful people. Koizumi Jun'ichirô était déjà connu pour être familier des

\footnotetext{
${ }^{10}$ En juin 2004, le Premier ministre dut se défendre devant la Diète d'allégations de viol commises quant il était encore étudiant, mais étouffées par son père, colportées par le tabloïd Nikkan gendai.
} 
plateaux de tournage, des milieux du cinéma, et des défilés de mode. Parmi les «tueuses » qu'il envoie contre les sortants PLD dissidents aux élections générales de 2005, figurent des célébrités habituées du petit écran telles que Fujino Makiko connue pour ses émissions culinaires, Katayama Satsuki une ancienne reine de beauté et top model, et une ancienne présentatrice de télévision, Koike Yuriko. Il se montre aux côtés d'acteurs hollywoodiens tels Richard Gere avec lequel il a une certaine ressemblance physique et Tom Cruise. Et quand il ne peut plus rencontrer ses chanteurs préférés, comme c'est le cas pour Elvis Presley, il se fait photographier aux côtés de sa veuve, Priscilla et de sa fille Lisa Marie à Memphis dans la propriété du King, lors de sa dernière visite aux Etats-Unis, en juin 2006, chaussé des mêmes mocassins de daim bleu qu'affectionnait son idole et des mêmes lunettes, esquissant quelques pas de danse. Il approche même en octobre 2005 Kitano Takeshi pour un poste ministériel. Kitano, avant d'être un metteur en scène connu internationalement, avait été l'un des amuseurs publics les plus en vue du petit écran. A l'instar des vedettes de la chanson, il commercialise des best of à son nom : c'est le cas de la compilation de ses chansons favorites d'Elvis Presley comportant sur la pochette un montage photographique avec le chanteur disparu. Il récidivera en octobre 2005, après son éclatante victoire aux élections générales, en publiant un second CD d'anthologie des musiques de film d'Ennio Morricone sous le label BMG Japan et dont le produit est redistribué à des institutions caritatives. Il dispose également de son propre album de photographies et pose aux côtés du Président Bush, le col ouvert et la balle de base-ball à la main. Si la peopolisation n'est pas en soi synonyme de proximité, car les people appartiennent par définition à un autre monde que celui du commun des mortels, le Premier ministre entend instaurer avec ses concitoyens le même type de relation qui existe entre une idole et son public : elle est à la fois inaccessible par sa position de star qui fait rêver, mais en même temps très proche par une gestion de l'image et des objets divers qui lui sont associés, qui permettent à la fois d'ancrer la star dans l'horizon quotidien de ses admirateurs, sans pour autant compromettre son statut transcendant.

Enfin on peut mettre au crédit de la peopolisation, les effets de mimétisme : son apparence détendue, - il lance en avril 2005 la mode " coolbiz », celle de vêtements légers, sans cravate - destinée à lutter contre l'utilisation systématique et excessive de l'air conditionné dans les bureaux dans le cadre des préoccupations environnementales du Protocole de Kyôto, et un an plus tard, sa ministre de l'Environnement, Mme Koike Yuriko lui propose, ainsi qu'aux ministres, de prendre part à un défilé de mode à Omote-sandô, l'un des quartiers chics de la capitale. Mais il s'agissait là de l'extension d'un parti pris de décontraction que les médias ont popularisé dès son arrivée au pouvoir en 2001. Prisant des costumes à la dernière mode italienne, le Premier ministre cultive aussi, avec ses pantalons Chinos et ses chemises Ralph Lauren, une image de «bourgeois bohème » à la japonaise qui le met en phase avec une population jeune, urbaine, "branchée », moins conformiste que les générations précédentes.

En bref, si peopolisation il y a, c'est moins dans l'étalage public de la vie privée que dans la « désacralisation » relative de l'homme public se mouvant dans des espaces virtuels de convivialité, à travers une succession de postures qui facilitent, pour l'homme de la rue, les processus d'identification et de reconnaissance.

\section{Conclusion}

La star Koizumi Jun'ichirô n'aura-t-elle été qu'une comète sans lendemain dans la vie politique japonaise ? Depuis son départ en septembre 2006, quatre mois après avoir été classé 
par le magazine Time parmi les 100 personnalités « qui ont fait bougé le monde » 1'ex Premier ministre a pratiquement disparu de la scène politique et le Japon a déjà usé deux chefs de gouvernement : Abe Shinzô, pourtant intronisé par son prédécesseur, n'avait pour lui que sa jeunesse et ses antécédents familiaux prestigieux, mais il se montra incapable de manipuler à son profit les outils institutionnels et médiatiques dont son prédécesseur avait habilement joué pour se maintenir au pouvoir. Avec Fukuda Yasuo, le Japon renoue avec les délices de la gérontocratie mais sans doute était-il nécessaire de stabiliser le jeu politique japonais après la « flamboyance » du mandat de Koïzumi Jun'ichirô et les déconvenues de son successeur, en renouant avec des formes plus conventionnelles de leadership. Il n'est pas certain cependant que l'expérience Koizumi ait été bénéfique pour le fonctionnement des institutions démocratiques dans son ensemble. Certes, l'opinion a pu être séduite par un style politique nouveau se démarquant à dessein des pratiques gouvernementales habituelles, ce qui prouve au moins que le citoyen japonais n'est pas fondamentalement hostile à une certaine forme d'incarnation du pouvoir. Mais il faut remarquer que ces phénomènes de médiatisation et de personnalisation du pouvoir tels qu'ils se sont développés en 1993 et dans le cadre du mandat de Koizumi ont été consécutifs à une perte à une perte de confiance à l'égard des institutions en général et du parti dominant en particulier, et que si la capacité de ce dernier à rebondir pour redresser des pratiques politiques pour le moins hétérodoxes est étonnante, les causes profondes de ce déficit de crédibilité demeurent. Ce qui l'a distingué indiscutablement de ses prédécesseurs, c'est la capacité non seulement à durer, mais à maintenir le système politique sous tension permanente en en contournant les pesanteurs. Quant à l'expérience même du gouvernement Koizumi, elle a fait l'objet d'appréciations pour le moins divergentes : pour les uns, elle marque une étape capitale vers la modernisation et la "maturation » de la vie politique nippone, en montrant que le volontarisme politique allié une stratégie efficace de communication et de réelles capacités de leadership peut venir à bout des résistances. D'autres en dénoncent les dérives démagogiques, l'abaissement induit du parlementarisme, la dictature de la médiacratie, l'appauvrissement du discours politique, voire une forme de dictature soft, de "fascisme souriant » fondée sur l'apathie des masses et la manipulation médiatique. Cette vision aporétique singularise à elle seule l'expérience Koizumi. Il reste à savoir désormais comment les électeurs japonais, séduits par l'homme, réagiront face au retour en force de formes plus convenues de gestion du politique et de gouvernance. 